\title{
Normative Vestibular-Ocular Reflex Gain Values for the Vertical Semicircular Canals
}

\author{
Louise Hag ${ }^{1}$, Emil Riis Abrahamsen ${ }^{2}$, Dan Dupont Hougaard ${ }^{2}$ \\ ${ }^{1}$ Aarhus University, Aarhus C; ${ }^{2}$ Department of Otolaryngology, Head \& Neck Surgery and Audiology, Aalborg University Hospital, Aalborg, Denmark
}

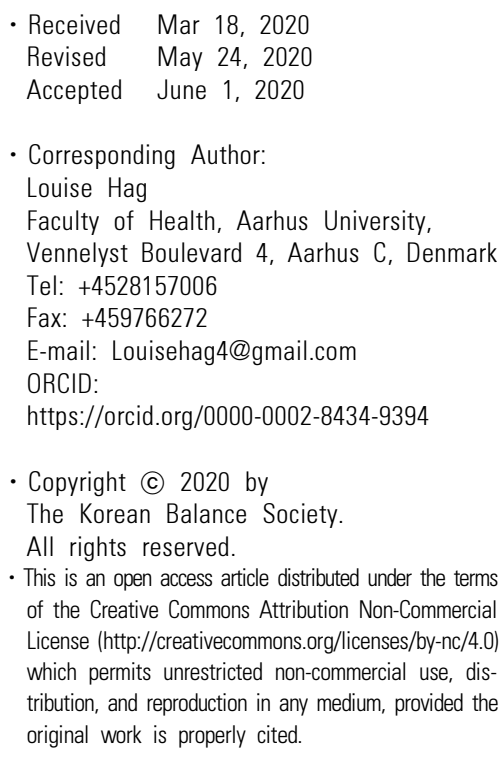

- This is an open access article distributed under the terms of the Creative Commons Attribution Non-Commercial License (http://creativecommons.org/licenses/by-nc/4.0) which permits unrestricted non-commercial use, distribution, and reproduction in any medium, provided the original work is properly cited.

Objectives: To determine normative vestibular-ocular-reflex (VOR) gain values for the vertical semicircular canals (SCCs) within a Danish cohort of healthy adults. Normative data regarding the vertical SCCs are sparse. Assessing the function of all 6 SCCs is important, as some vestibular diseases may selectively affect specific SCCs.

Methods: A prospective cross-sectional study of 60 subjects aged 18-65 years with no previous vestibular disorders. All subjects underwent complete examination of all 6 SCCs with EyeSeeCam video head impulse test (vHIT) system. Results: Mean VOR gain values of the right anterior and left posterior canal were 1.46 and 1.43 respectively. For the left anterior and right posterior canals, mean VOR gain values were 0.96 and 0.97 respectively.

Conclusions: Our results suggest that mean VOR gain is close to 1.4 in the right anterior and left posterior plane and close to one for the left anterior and right posterior plane when testing the vertical SCCs in healthy adults with the EyeSeeCam vHIT system.

Res Vestib Sci 2020;19(2):62-70

Keywords: Normative data; Video head impulse test; VOR gain; Vestibular testing; EyeSeeCam

\section{INTRODUCTION}

The video head impulse test (vHIT) is a quick and simple test for the examination of vestibular function based on the principal of the vestibular-ocular-reflex (VOR). The test is adapted from the clinical head impulse test, first described in 1988 [1]. Two decades later, in 2009, the vHIT system was first demonstrated to be a valid tool for testing the function of the horizontal [2,3] and later, in 2013, the vertical semicircular canals (SCCs) [4,5].

The vHIT system measures eye movement responses to abrupt, brief, fast, and unpredictable head turns (termed head impulses) with low amplitudes and high accelerations. When the head of a subject is turned passively, while maintaining fixation on a target, the eyes will, reflectively, move in the opposite direction of the head movement. This enables the gaze to remain unperturbed on the target with a latency of only $8 \mathrm{msec}$. In healthy subjects, 3 groups of oculomotor reflexes utilize gaze fixation: the optokinetic, cervico-ocular, and vestibular-ocularreflex. If the head is turned quickly (faster than $150^{\circ} / \mathrm{sec}$ ) the optokinetic and cervico-ocular reflexes will be too slow to be activated, and the healthy subject will instead make compensatory fast eye movements (i.e., saccades) to stay on target solely by means of the VOR. Therefore, moving the head passively and briskly in the planes of the SCCs, while measuring the compensatory eye movements, enables the examiner to test the VOR exclusively as an indicator of SCC function [1,6].

The quantification of the VOR function is measured in terms of a "gain" value. VOR gain is calculated as the ratio of the compensatory eye peak velocity to head peak velocity and is, 
in healthy individuals, approximately one. Gain calculation methods are predefined by the accompanying company software, as different vHIT systems calculate gain values in quite different ways. Currently no golden standard gain calculation method exists for vHIT [7].

Several studies have collected normative data on gain values for the horizontal SCCs [8-12] but quantitative data on normative VOR gain values for the vertical SCCs are sparse and do not exist for this vHIT system [9,10]. Examination of the vertical SCCs is important, as some vestibular disorders may selectively involve certain SCCs. However, examination of the vertical SCCs may be a challenging procedure as vertical head impulses are more technically demanding to deliver. Additionally, the compensatory eye movements following these head impulses include a torsional component, which the vHIT system is yet not able to detect [4]. Nevertheless, the uncertainty related to the test procedure and the subsequent interpretation of the results merely emphasizes the importance of well-founded studies providing normative values of VOR gain for the vertical SCCs.

The aim of this study was to identify normative values of VOR gain for the vertical semicircular canals within a Danish cohort of healthy adults assessed by use of the vHIT. In addition, advantages and disadvantages of vertical semicircular canal testing are evaluated in order to determine whether or not these additional tests should be implemented in future examinations of vertiginous adults.

\section{MATERIALS AND METHODS}

\section{Study Design}

This study was conducted as a prospective cross-sectional study [13].

\section{Subjects}

Sixty subjects (age range, 18 to 65 years; mean \pm standard deviation, $42.6 \pm 13.9$ years) with no prior history of vestibular disease were enrolled in this study. All subjects were recruited and tested at the Department. Subjects recruited included subjects either scheduled for a regular appointment at the Depart- ment, relatives or staff. See Table 1 for population characteristics. Informed consent was obtained from all the subjects who participated in the study. The study was approved by the North Denmark Region Committee on Health Research Ethics (reference number: N-20160062). All procedures performed in this study were in accordance with the ethical standards of the 1964 Helsinki declaration and its later amendments or comparable ethical standards.

Inclusion criteria were limited to an age range between 18 and 65 years of age as well as no previously known vestibular disorder(s). Prior to inclusion, all subjects were asked to fill out the Dizziness Handicap Inventory (DHI) with a total score of 15 or above leading to exclusion. Hence, a score below 15 was considered equal to having no dizziness handicap [14]. Finally, all subjects were tested with video Frenzel Goggles to rule out any spontaneous and/or gaze-induced nystagmus as well as compromised eye muscle motilities and/or eye muscle palsies. Any pathological findings also led to exclusion.

In total 156 subjects were assessed for eligibility. 36 subjects were excluded (reasons specified below) and, following randomization, half of the 120 included subjects (60 subjects) were allocated to an additional vHIT study. Seventeen subjects were excluded due to previous known vestibular disorder(s), 6 subjects because of previous ear surgery, 3 subjects due to known central nervous system disorder and 2 subjects due to previous sudden hearing loss. Four subjects were unable to cooperate during examination and lastly, 4 subjects were excluded due to a DHI-score above 15 . Sixty subjects were randomized to

Table 1. Population characteristics $(n=60)$

\begin{tabular}{lc}
\hline \multicolumn{1}{c}{ Characteristic } & Value \\
\hline Age $(\mathrm{yr})$ & $42.6 \pm 13.9$ \\
Sex & $36(60.0)$ \\
$\quad$ Female & $24(40.0)$ \\
Male & \\
DHI scores & $0.73(0-8)$ \\
$\quad$ Physical & $0.23(0-8)$ \\
Functional & $0.23(0-8)$ \\
Emotional & $1.19(0-14)$ \\
Total score &
\end{tabular}

Values are presented as mean \pm standard deviation, number (\%), or mean (range).

DHI, Dizziness Handicap Inventory. 
testing with an additional vHIT system for another study.

\section{Software System}

All subjects were tested with the EyeSeeCam vHIT system (Interacoustics, Middelfart, Denmark).

\section{Experimental Procedures}

All subjects were tested in the same well-lit room. Markings on the floor ensured that all subjects were tested in the same position. The markings were placed at a distance of 1.5 meters from the wall in accordance with the manufacturer's recommendations.

All subjects underwent 4 separate tests of all vertical SCCs. The same 2 examiners performed all tests. Examiner 1 was a senior Ear Nose and Throat specialist with several years of experience with vHIT testing while examiner 2 was a medical student with only sparse pretrial practice. Each examiner completed 2 tests with each subject. Pretest randomization determined order of SCC testing as well as initial examiner.

The EyeSeeCam system consists of a high-speed digital camera $(220 \mathrm{~Hz})$ and a gyroscope mounted on a lightweight goggle. It is possible to mount the camera on either side of the goggle, but it was placed on the right side as default. OtoAccess (Interacoustics) software version 1-2-1 was used with the vHIT system.

The goggles were tightened firmly before each test to avoid goggle slippage [15]. A standard calibration preceded all separate tests. Following calibration, head impulses were performed until 15 head impulses for each of the vertical SCCs were accepted by the accompanying software. To perform a head impulse, the examiner placed his right (dominant) hand on top of the head, and the left (other) hand below the chin of the subject. The head was then moved fast, unpredictably, with low amplitude, and with high acceleration in the plane of the vertical SCCs which is approximately $45^{\circ}$ to the sagittal plane of the head [16] while the subject maintained fixation at an earth fixed target. This means that, for assessment of the function of the vertical SCCs, the head is rotated right-downward to left-upward in the plane of the right anterior and left posterior canals (RALP) or left downward to right-upward in the plane of the left anterior and right posterior canals (LARP). The accompanying software provides a guide that shows if the vertical head impulses are made in the correct planes. A line is plotted after each head impulse depicting the angle measured for that thrust. Lines within a shaded area shows if the direction of the head impulse is within tolerance for an acceptable impulse angle. Head- and eye velocities must range between $100^{\circ}$ to $310^{\circ}$ per second and head acceleration must exceed $1000^{\circ} / \mathrm{sec}^{2}$ to be detected as a valid head impulse [17].

See Fig. 1 for illustration of the test procedure. During the test, the examiners were careful not to touch the goggles to avoid infliction of any noise and/or artifacts. The range of peak head velocities for accepted head impulses was set between $100^{\circ}$ and $310^{\circ}$ per second for all 4 vertical SCCs (software

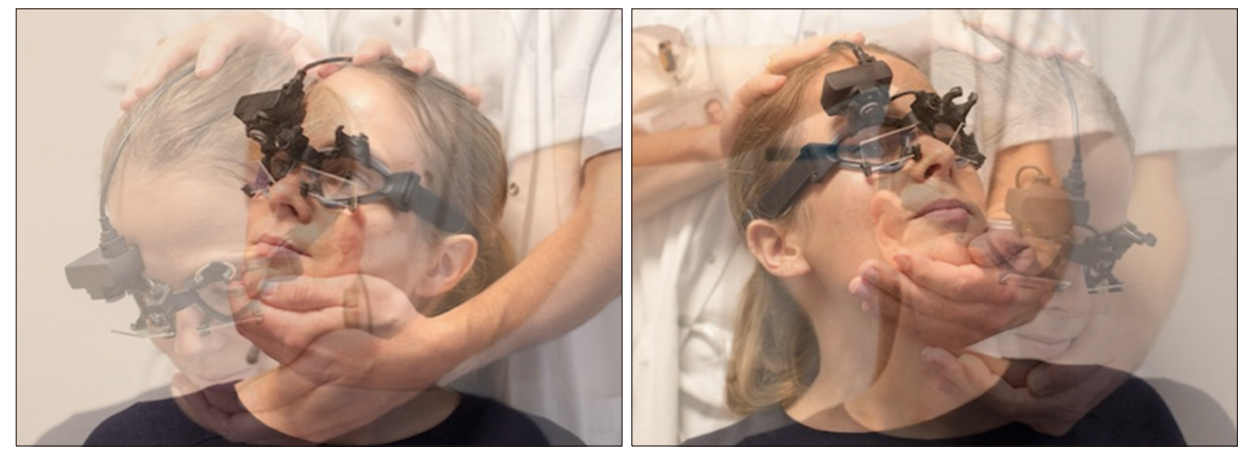

Fig. 1. Visualization of the 3-dimensional video head impulse test (vHIT) procedure with the EyeSeeCam vHIT system for vertical semicircular canals (SCC) testing. The examiner placed his dominant hand on top of the head and the other hand beneath the chin. The head was then rotated in the direction of the SCC being tested. Left side illustrates right anterior and left posterior SCC testing and right side illustrates left anterior and right posterior SCC testing. 
default setting). Additionally, acceptance of the individual head impulse requires that the peak head velocity must appear no later than $80 \mathrm{msec}$ into the head thrust. Upon completion of the test, the collected head impulses underwent automatic data selection by the accompanying software using algorithms predefined by the manufacturer. The experienced examiner then performed manual data selection with removal of both artifacts and noise, aiming to reach at least 10 artifact-free head impulses for each SCC [10]. Impulses were deleted if the traces displayed obvious artifacts and/or noise.

The accompanying OtoAccess software calculated regression VOR gain values for the vertical SCCs. Regression VOR gain was perceived to be less susceptible to sources of errors compared to gain values based on few data points [4]. With regression gain, eye velocities are described as a function of head velocities. The EyeSeeCam protocol calculates the vertical VOR gain as the regression slope between the vertical components of head- and eye velocities. Analysis is restricted to head- and eye velocity data pairs collected between 0 and $100 \mathrm{msec}$ after initiation of the actual head impulse to avoid inclusion of data from extra vestibular signals. Regression gain is calculated as an average of the eye-to-head speed ratio from 0 to $100 \mathrm{msec}$. Through interpolation, the $220-\mathrm{Hz}$ camera is up-scaled to 1000 Hz. This provides data from 100 measure points where the eye velocity in each of the new head impulses is paired with the corresponding head velocity. These "velocity sets" are then plotted in the regression diagram and a straight line is plotted with a slope corresponding to the average of the measured velocity set. The collected data sets are used to calculate and display the regression slope as a measure of VOR gain. The formula used for calculation of the regression slope adds eye velocity values over time and multiplies these values with the corresponding head velocity values (numerator). The number is then divided by the square of head velocity values over time (denominator). The regression plots provided allows graphical data analysis over the entire velocity range of the performed head impulses and is shown as a best-fitted line through these data pairs [18-20].

Saccades were defined as pathological if all the following criteria were met:

(1) The saccades appeared in more than $50 \%$ of the head impulses.

(2) The amplitude of the saccadic eye movement had to be at least half of the velocity of the head movement.

(3) Saccades must present in the opposite direction of the head movement.

(4) The saccades must occur from approximately $100 \mathrm{msec}$ from the onset of the head movement to approximately $100 \mathrm{msec}$ after the head movement has stopped [13].

\section{Statistical Analysis}

All clinical data were deposited in the Redcap database (Redcap, Vanderbilt University, Nashville, TN, USA) and all statistical analysis was performed with STATA (StataCorp LLC, College Station, TX, USA). The data included regression gain values calculated by the appertaining software system. Since all subjects underwent 4 separate tests of all vertical SCCs, mean VOR gain values and standard deviations were calculated from the mean VOR gain of all 4 intrasubject tests, as mean values from multiple tests are considered to provide a more reliable result.

\section{RESULTS}

All 60 subjects included in this study underwent complete vHIT examination. Mean number of impulses are outlined in Table 2.

Main results are presented in Tables 3 and 4. For a complete set of data refer to Supplementary materials 1 and 2. Mean VOR gain values of the sorted RALP are 1.46 and 1.43 respectively, while the corresponding values of the sorted LARP were 0.96 and 0.97 . 'Sorted' impulses include all remaining

Table 2. Experimental data

\begin{tabular}{lc}
\hline \multicolumn{1}{c}{ No. of head impulses } & Mean \pm SD \\
\hline Right anterior & $11.50 \pm 2.62$ \\
Left posterior & $11.45 \pm 2.71$ \\
Left anterior & $11.29 \pm 2.77$ \\
Right posterior & $11.00 \pm 2.98$ \\
\hline
\end{tabular}

Number of head impulses applied to each of the vertical semicircular canals.

SD, standard deviation. 
Res Vestib Sci Vol. 19, No. 2, Jun. 2020

Table 3. Mean gain values for the vertical semicircular canals

\begin{tabular}{lcccccccc}
\hline & ra & ra (sort) & lp & lp (sort) & la & la (sort) & rp & rp (sort) \\
\hline Mean & 1.46 & $\mathbf{1 . 4 6}$ & 1.44 & $\mathbf{1 . 4 3}$ & 0.95 & $\mathbf{0 . 9 6}$ & 0.96 & $\mathbf{0 . 9 7}$ \\
95\% CI & $1.38-1.54$ & $1.38-1.54$ & $1.34-1.54$ & $1.34-1.53$ & $0.90-1.00$ & $0.91-1.01$ & $0.91-1.02$ & $0.92-1.02$ \\
SD & 0.32 & 0.31 & 0.38 & 0.37 & 0.19 & 0.19 & 0.20 & 0.19 \\
\hline
\end{tabular}

Mean gain values of all 4 vertical semicircular canals for all video head impulse test examinations before and after (sort) manual data selection. Mean gain values of the sorted data for the right anterior and left posterior and left anterior and right posterior planes are shown in bold. Note that the difference between sorted and unsorted mean gain values are all insignificant and the very high mean gain values of the right anterior and left posterior semicircular canals.

ra, right anterior; sort, sorted impulses; lp, left posterior; la, left anterior; rp, right posterior; CI, confidence interval; SD, standard deviation.

Table 4. Range and median values of VOR gain for the vertical semicircular canals

\begin{tabular}{lcccccccc}
\hline & ra & lp & la & rp & ra (sort) & lp (sort) & la (sort) & rp (sort) \\
\hline Low & 0.21 & 0.33 & 0.11 & 0.15 & 0.21 & 0.38 & 0.04 & 0.08 \\
High & 2.86 & 3.48 & 1.86 & 1.97 & 2.91 & 3.39 & 2.01 & 1.95 \\
Median & 1.42 & 1.37 & 0.94 & 0.93 & 1.42 & 1.36 & 0.96 & 0.94 \\
\hline
\end{tabular}

Range and median gain values of all 4 vertical semicircular canals for all video head impulse test examinations before and after (sort) manual data selection.

VOR, vestibulo-ocular reflex; ra, right anterior; lp, left posterior; la, left anterior; rp, right posterior; sort, sorted impulses; Low, lowest range value; High, highest range value; Median, median value.
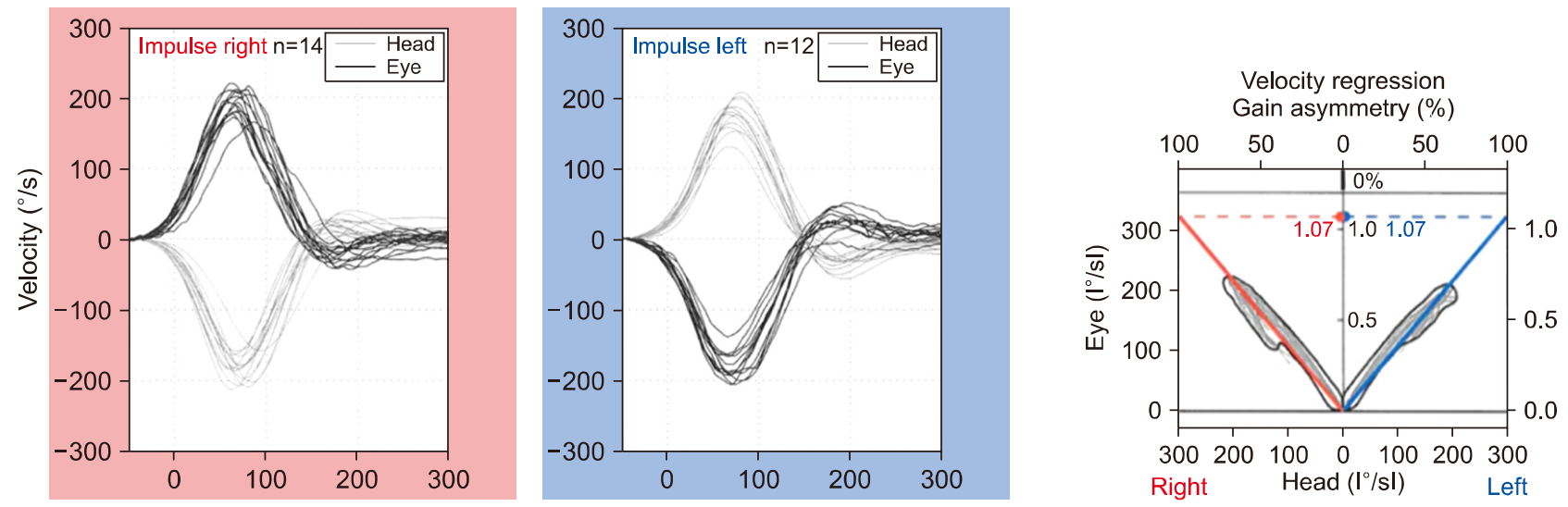

Fig. 2. Test report following horizontal semicircular canals (SCC) testing of a healthy subject. Please note that the graphs depicting head- and eye velocities are symmetrical and that concomitant regression gain values are within the normal range for both the left and right horizontal SCCs.

head impulses following manual data selection by the experienced examiner as described in the previous section. Solely the algorithms incorporated in the accompanying software have processed the "unsorted" head impulses. Mean VOR gain values before manual data selection were 1.46 and 1.44 for the RALP respectively. For the left anterior and right posterior SCCs the corresponding mean VOR gain values were 0.95 and 0.96 . The results show that the mean gain VOR values of the sorted and unsorted head impulses are highly comparable, as the differ- ences do not exceed 0.01 . Note that the asymmetry in the test results is specific for the vertical SCCs compared to horizontal SCC testing. For clarity, a VOR gain result of horizontal SCC testing is depicted in Fig. 2.

Pathological saccades were only found in one subject, and only in vHIT examinations performed by examiner 2 . The saccades were found in the left posterior SCC in both examinations with accompanying mean gain values of 0.96 and 1.38 . 


\section{DISCUSSION}

The results from the present study suggest that mean VOR gain values are approximately 1.4 and 1.0 for the RALP and LARP planes respectively, when testing healthy adults between 18 and 65 years of age using the EyeSeeCam vHIT system. These findings are seemingly counterintuitive, as values of gain, theoretically, is one or lower. Furthermore, the results are not in line with previous studies, suggesting that normative mean VOR gain is below one for both the RALP and LARP planes $[9,10,21]$. However, these studies are based on data obtained using different vHIT systems with separate gain calculation methods. To the best of our knowledge, no previous study has collected normative vertical VOR gain data using the EyeSeeCam system. The results in this study therefore rise 2 important questions that will be discussed in the following sections.

(1) Why were "normal" mean gain values higher than expected with vertical SCC testing?

The higher than expected mean gain values may be a consequence of imprecise measurements as the EyeSeeCam vHIT system originally was created for horizontal SCC testing. Compensatory eye movements to vertical canal stimulation by the 3-dimensional vHIT test method usually contain a torsional component, which the vHIT system is not able to detect. To our knowledge, no previous study has validated vertical SCC testing with the EyeSeeCam vHIT system by comparing it with the scleral search coil technique, which is regarded as the golden standard test.

VOR gain values may also be modified by multiple possible pitfalls during examination. There are a lot of moving parts (strap, goggle, and camera), and they do not always stick together. When the head is thrust one direction, the camera tries to stay behind due to inertia, and the accompanying eye velocity may be wrong as the camera bounces around on the face. When severe, this can cause a problem where the eyes and the head are not aligned in time. They come together at the end and presumably, this happens because the head and goggles separate during the head impulse and then rejoin immediately after completion of the head impulse. Usually, this will cause a too high gain problem. Other contributing factors may include loose skin, slippery hair, involuntary head movements, or inability to relax during impulses. Artifacts may also be a consequence of improper targeting of the pupil by the eye-tracking software [22]. Finally, if the head impulses are performed too quickly, e.g., faster than $250^{\circ}$ per second, it may influence the examination by causing a lot of noise, leading to high gain values [23].

Furthermore, gaze direction has a major effect on the measured VOR when testing the vertical canals, because the head impulses will start to produce a roll response, that is not detectable, as the gaze drifts out of the plane of stimulus [6]. Therefore, it is important, that gaze remains as close to the stimulated head plane as possible. Finally, it should be noticed, that the distance to the target is of importance. Thus, if the patient is seated too close to the marking, the eyes will conjugate affecting the reliability of the measured gain values. In this study however, subjects were placed at a distance of 1.5 $\mathrm{m}$ from the wall in accordance with the manufacturer's recommendations.

Surely, there are various possible pitfalls, when performing the vHIT examination. These factors are more likely to occur, if the test is not performed in accordance with the predefined standards of the specific software system. With this study, however, these factors are not considered a major problem, since all standard procedures were followed thoroughly. Additionally, the experienced examiner evaluated and interpreted all vHIT reports, ensuring high quality of individual tests and low occurrence of noise and/or artifacts. Due to this clinical evaluation, one may question whether the test truly can be categorized as an entirely objective test. However, our results suggest that manual data selection does not significantly affect the test results with this vHIT system, since mean gain values before and after manual data selection are closely comparable.

(2) Why were mean RALP gain values higher than mean LARP gain values?

A recent study has shown that the placement of the camera influences gain values, as slightly higher gain values were reported on the camera-holding side when testing the horizontal canals [24]. In vHIT testing, the camera is eccentric to the center, so if you rotate the head through the plane of the camera, it will bounce around even more. In this study, the camera was placed on the right side as default. One can only speculate, if this might have been a contributing factor to the 
abnormally high gain values seen with RALP testing.

Interestingly, both examiners were right-handed. During leftward displacements, the right-hand leads the action of movement, which possibly makes it easier to perform compared to rightward impulses. Therefore, the higher VOR gain values measured during rightward head impulses may be a consequence of the right-handedness of the examiners [11,25]. Another tentative, yet possible, explanation may be found within the hardware of the EyeSeeCam system, supposing that VOR gain values of the RALP plane are systematically overestimated because of possible sources of errors in the underlying settings of the system.

When taking the following into consideration, the authors of this article believe that the very high (nonphysiological) mean gain values seen with RALP testing, for the most part, has to be related to technical issues: all individual steps of standard procedures were followed meticulously in accordance with the manufacturer's recommendations. Two examiners, reducing the risk of systematic technical errors, performed all tests. Originally, the hardware, together with the accompanying software, was developed for horizontal SCC testing only, and, importantly, scleral search coil validation for the vertical SCCs is missing. The right-handedness of the examiners and the placement of the camera might have contributed to the very high gain mean values seen with RALP testing and, therefore, consideration of these issues is recommended when performing vertical head impulses prospectively. Nevertheless, it is the opinion of the authors that the reasons listed here cannot explain the very high mean gain values seen with RALP testing. However, this remains speculations, and further investigations are needed in order to clarify possible underlying mechanisms.

In conclusion, it may be said that gain values always should be evaluated in context with the presence or absence of pathological saccades. In this study however, pathological saccades were found in only 1 out of 60 subjects, and only in the vHIT examinations performed by examiner 2 ( 2 out of 960 individual SCC tests). Since the saccades were not present in the examinations performed by examiner 1 (the experienced examiner), it can be speculated, if these saccades represented a technical artifact, possibly linked to the examiners' level of experience. Evidently, one would expect that truly pathological saccades would be present in all impulses regardless of the examiner.
Interestingly, a study testing 2 separate vHIT systems revealed some level of interexaminer variability between 2 examiners when using the EyeSeeCam system for examination [13]. The authors therefore conclude that some level of experience is beneficial when performing the vHIT examination with this vHIT system.

\section{Limitations of the Study}

Subjects were recruited at the Department of Otolaryngology, Head \& Neck Surgery and Audiology, Aalborg University Hospital. This meant that subjects either were patients scheduled for a regular appointment, relatives to these or staff. This might cause potential selection bias, consequently affecting the generalizability of the results. However, subjects were thoroughly examined prior to inclusion in order to rule out any vestibular pathologies or eye movement abnormalities that might have affected the results. Furthermore, it is important to underline that the results represent data from one single vHIT system. Therefore, no conclusions regarding other vHIT systems can be made. The authors of this article are aware that, theoretically, a gain value above one must be considered nonphysiological. However, this study was designed and carried out in such a way, that all test procedures were done in meticulous accordance with the manufacturer's recommendations. This means that all known factors potentially capable of altering the mean gain values have been known and taking into consideration by both examiners before (pretest conditions), during (test procedures) and after (manual data selection) the actual testing.

\section{Clinical Importance}

Measuring the function of all 6 SCC enables the physician to refine the clinical diagnosis, as some vestibular lesions may selectively involve certain SCCs. In vestibular neuritis (VN), for example, it is possible that the entire vestibular nerve or only a part of it is affected. Involvement of only the horizontal and anterior SCCs indicate the diagnosis of 'classic' superior $\mathrm{VN}$, whereas isolated loss of posterior SCC function on the other hand indicates the diagnosis of selective inferior VN [26]. Similarly, in vestibular schwannoma (VS), certain parts of the 
vestibular nerve may be affected. A retrospective study found, that inferior vestibular nerve tumors were twice as common as superior nerve tumors [27]. Additionally, the nerve of origin in VS is an important prognostic factor for hearing preservation surgery [28]. Moreover, vertical SCC testing helps evaluating superior canal function in patients with superior canal dehiscence syndrome [29]. In bilateral vestibulopathy (BVP), quantifying vHIT gain for all 6 SCCs may help identifying the underlying disorder, as different etiologies show disease-specific patterns of SCC impairment [30]. Finally, it aids differentiating central lesions with bilateral affection of SCC function from BVP [31]. However, this study suggests that the examiner must apply higher than expected normative gain values with RALP testing; bearing in mind that, for instance, a gain value of one may therefore indicate hypofunction of either of these 2 SCCs.

In conclusion, results from our study suggest that mean VOR gain values of the right anterior and left posterior plane are 1.46 (95\% confidence interval [CI], 1.38-1.54) and 1.43 (95\% CI, 1.34-1.53) respectively. The mean VOR gain values of the left anterior and right posterior plane are 0.96 (95\% CI, 0.91-1.01) and 0.97 (95\% CI, 0.92-1.02) respectively, when testing a population of healthy Danish adults between 18-65 years of age using the EyeSeeCam vHIT system. No previous study has provided normative data for comparison, so future investigations are needed in order to substantiate our findings.

Despite the complexity, vertical canal testing with the vHIT system proved to be a well-tolerated and highly applicable procedure with many advantages. We therefore recommend, that vertical SCC testing should be included as part of the standard vHIT examination of adults. However, vertical SCC test results need to be validated against the scleral search coil technique before unequivocal conclusions on vertical SCC function can be made with this vHIT system.

\section{CONFLICT OF INTEREST}

No potential conflict of interest relevant to this article was reported.

\section{SUPPLEMENTARY MATERIALS}

Supplementary material can be found via https://doi.org/10. 21790/rvs.2020.19.2.62.

\section{REFERENCES}

1. Halmagyi GM, Curthoys IS. A clinical sign of canal paresis. Arch Neurol 1988;45:737-9.

2. Weber KP, MacDougall HG, Halmagyi GM, Curthoys IS. Impulsive testing of semicircular-canal function using video-oculography. Ann N Y Acad Sci 2009;1164:486-91.

3. MacDougall HG, Weber KP, McGarvie LA, Halmagyi GM, Curthoys IS. The video head impulse test: diagnostic accuracy in peripheral vestibulopathy. Neurology 2009;73:1134-41.

4. Macdougall HG, McGarvie LA, Halmagyi GM, Curthoys IS, Weber KP. The video Head Impulse Test (vHIT) detects vertical semicircular canal dysfunction. PLoS One 2013;8:e61488.

5. MacDougall HG, McGarvie LA, Halmagyi GM, Curthoys IS, Weber KP. Application of the video head impulse test to detect vertical semicircular canal dysfunction. Otol Neurotol 2013;34: 974-9.

6. Halmagyi GM, Chen L, MacDougall HG, Weber KP, McGarvie LA, Curthoys IS. The Video Head Impulse Test. Front Neurol 2017;8:258.

7. Cleworth TW, Carpenter MG, Honegger F, Allum JHJ. Differences in head impulse test results due to analysis techniques. J Vestib Res 2017;27:163-72.

8. Mossman B, Mossman S, Purdie G, Schneider E. Age dependent normal horizontal VOR gain of head impulse test as measured with video-oculography. J Otolaryngol Head Neck Surg 2015;44:29.

9. Bansal S, Sinha SK. Assessment of VOR gain function and its test-retest reliability in normal hearing individuals. Eur Arch Otorhinolaryngol 2016;273:3167-73.

10. McGavie LA, MacDougall HG, Halmagyi GM, Burgess AM, Weber KP, Curthoys IS. The video head impulse test (vHIT) of semicircular canal function - age-dependent normative values of VOR gain in healthy subjects. Front Neurol 2015;6:154.

11. Matiño-Soler E, Esteller-More E, Martin-Sanchez JC, MartinezSanchez JM, Perez-Fernandez N. Normative data on angular vestibulo-ocular responses in the yaw axis measured using the video head impulse test. Otol Neurotol 2015;36:466-71.

12. Blödow A, Pannasch S, Walther LE. Detection of isolated covert saccades with the video head impulse test in peripheral vestibular disorders. Auris Nasus Larynx 2013;40:348-51.

13. Abrahamsen ER, Christensen AE, Hougaard DD. Intra- and interexaminer variability of two separate video head impulse test systems assessing all six semicircular canals. Otol Neurotol 2018;39:e113-22.

14. McCaslin DL. Electronystagmography and Videonystagmography. San Diego (CA): Plural Publishing; 2013.

15. Migliaccio AA, Cremer PD. The 2D modified head impulse 
test: a 2D technique for measuring function in all six semi-circular canals. J Vestib Res 2011;21:227-34.

16. Bradshaw AP, Curthoys IS, Todd MJ, Magnussen JS, Taubman DS, Aw ST, et al. A mathematical model of human semicircular canal geometry: a new basis for interpreting vestibular physiology. J Assoc Res Otolaryngol 2010;11:145-59.

17. EyeSeeCam. Instructions for use - EyeSeeCam vHIT - part 1 (Nov 9, 2016) and part 2 (Dec 1, 2016). Middelfart (Denmark): Interacoustics $\mathrm{A} / \mathrm{S}$; c2020. Available from: https://www. interacoustics.com/balance/software/eyeseecam.

18. Gain calculation methods in vHIT - Interacoustics Academy [Internet]. YouTube; 2017. Available from: https://www. youtube.com/watch? $=$ =OYs_gilca2E.

19. Aw ST, Haslwanter T, Halmagyi GM, Curthoys IS, Yavor RA, Todd MJ. Three-dimensional vector analysis of the human vestibuloocular reflex in response to high-acceleration head rotations. I. Responses in normal subjects. J Neurophysiol 1996; 76:4009-20.

20. Aw ST, Halmagyi GM, Haslwanter T, Curthoys IS, Yavor RA, Todd MJ. Three-dimensional vector analysis of the human vestibuloocular reflex in response to high-acceleration head rotations. II. responses in subjects with unilateral vestibular loss and selective semicircular canal occlusion. J Neurophysiol 1996;76:4021-30.

21. Cremer PD, Halmagyi GM, Aw ST, Curthoys IS, McGavie LA, Todd MJ, et al. Semicircular canal plane head impulses detect absent function of individual semicircular canals. Brain 1998; 121(Pt 4):699-716.

22. Mantokoudis G, Saber Tehrani AS, Kattah JC, Eibenberger K, Guede CI, Zee DS, et al. Quantifying the vestibulo-ocular reflex with video-oculography: nature and frequency of artifacts. Audiol Neurootol 2015;20:39-50.

23. Kim TS, Lim HW, Yang CJ, Kim YH, Choi WR, Kim YR, et al. Changes of video head impulse test results in lateral semicircular canal plane by different peak head velocities in patients with vestibular neuritis. Acta Otolaryngol 2018;138: 785-9.

24. Strupp M, Kichler A, McGarvie L, Kremmyda O. The video head impulse test: a right-left imbalance. J Neurol 2018;265 (Suppl 1):40-3.

25. Patterson JN, Bassett AM, Mollak CM, Honaker JA. Effects of hand placement technique on the video head impulse test (vHIT) in younger and older adults. Otol Neurotol 2015; 36:1061-8.

26. Aw ST, Fetter M, Cremer PD, Karlberg M, Halmagyi GM. Individual semicircular canal function in superior and inferior vestibular neuritis. Neurology 2001;57:768-74.

27. Jacob A, Robinson LL Jr, Bortman JS, Yu L, Dodson EE, Welling DB. Nerve of origin, tumor size, hearing preservation, and facial nerve outcomes in 359 vestibular schwannoma resections at a tertiary care academic center. Laryngoscope 2007; 117:2087-92.

28. Constanzo F, Sens P, Teixeira BCA, Ramina R. Video head impulse test to preoperatively identify the nerve of origin of vestibular schwannomas. Oper Neurosurg (Hagerstown) 2019; 16:319-25.

29. Carey JP, Migliaccio AA, Minor LB. Semicircular canal function before and after surgery for superior canal dehiscence. Otol Neurotol 2007;28:356-64.

30. Tamutzer AA, Bockisch CJ, Buffone E, Weiler S, Bachmann LM, Weber KP. Disease-specific sparing of the anterior semicircular canals in bilateral vestibulopathy. Clin Neurophysiol 2016;127:2791-801.

31. Chen L, Halmagyi GM. Central lesions with selective semicircular canal involvement mimicking bilateral vestibulopathy. Front Neurol 2018;9:264. 